Check for updates

Cite this: RSC Adv., 2019, 9, 9435

Received 23rd January 2019

Accepted 15th March 2019

DOI: $10.1039 / c 9 r a 00590 k$

rsc.li/rsc-advances

\section{Amino-polystyrene supported hexaethylene glycol-bridged ionic liquid as an efficient heterogeneous catalyst for water-mediated nucleophilic hydroxylation $\uparrow$}

\author{
Mudumala Veeranarayana Reddy, Seok Min Kang, Suah Yoo, Sang Sik Woo \\ and Dong Wook Kim (D) *
}

\begin{abstract}
We report a simple and eco-friendly method for producing an amino-polystyrene supported hexaethylene glycol-bridged ionic liquid (APS-HEGBIL) based on the copolymerization of amino-styrene with 1-vinyl imidazolium ionic liquid bearing hexaethylene glycol moieties, and its characterization by several analytical techniques. The resulting APS-HEGBIL catalyst was found to be remarkably efficient at catalyzing the selective nucleophilic hydroxylation of alkyl halides to produce the corresponding alcohols in water, which acted as a solvent and a nucleophilic hydroxide source. The catalyst was easily recycled and maintained its catalytic activity and stability after ten cycles with excellent yields. The main attributes of the catalyst were that it significantly enhanced the nucleophilicity of water during reactions and promoted the rapid conversions of polar and base-sensitive alkyl halide reactants to alcohols in excellent yields. The combination of ionic liquids and polymeric materials afforded quasi-homogeneous catalysts that were recycled by simple filtration and provided environmentally benign means for conducted catalytic procedures.
\end{abstract}

\section{Introduction}

Currently, the synthesis of organic compounds by utilizing improved environmentally benign catalytic systems and conditions is an aspiration of organic chemists. ${ }^{1}$ The sustainability of green chemistry depends on; (i) the use of multicomponent reactions, solvent-free conditions, or benign solvents, (ii) the discovery of promising, less toxic reagents and catalytic systems, (iii) the design of reliable and cost-effective methodologies, and (iv) the use of renewable resources, energy efficient catalytic processes, and the further development of separation procedures. $^{2,3}$ With these objectives in mind, considerable interest has been focused on ionic liquids (ILs) during the past decade because they can provide efficient media and catalysts for organic reactions (Fig. 1). ${ }^{4,5}$ The attractive properties of ILs include ease of separation, recyclability, and immobilization on heterogenous solid supports, ${ }^{6-9}$ and the combined advantages of environmentally friendly heterogeneous catalysis and sustainable catalytic properties of polymer supported ionic liquids (PSILs) have resulted in their being recognized as ranking among the best green catalytic systems. Furthermore, the heterogenization of active molecules to aid the isolation and

Department of Chemistry and Chemical Engineering, World Class Smart Laboratory (WCSL), Inha University, 100 Inha-ro, Nam-gu, Incheon 402-751, Korea. E-mail: kimdw@inha.ac.kr; Fax: +82-32-867-5604; Tel: +82-32-860-7679

$\dagger$ Electronic supplementary information (ESI) available. See DOI: 10.1039/c9ra00590k separation of catalysts has resulted in many attempts to utilize PSILs in various organic transformations over the last two decades. ${ }^{10-13}$

On the other hand, environmental issues have steered research in the catalytic field towards environmentally friendly reaction media, such as water as a solvent/co-solvent. ${ }^{14,15}$ In addition to the low cost of water and its availability and nontoxic nature, its high polarity, ability to form hydrogen bonding networks, high specific heat capacity, high cohesive energy, and large surface tension significantly influence organic conversions. ${ }^{16-18}$

A diverse range of biologically relevant molecules contain the hydroxyl group, and thus, alcohols constitute important

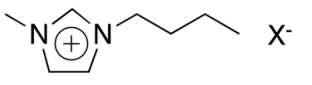

[bmim][OMs] $\left(\mathrm{X}=\mathrm{OMs} \mathrm{BF}_{4}\right)$ as a conventional IL
Fig. 1 Conventional and custom-made ionic liquids. bmim $=1-n-$ butyl-3-methylimidazolium; hexaEGmim = 1-hexaethylene glycol 3methylimidazolium; hexaEG-DHIM = hexaethylene glycol-bridged dicationic imidazolium dimesylate. 


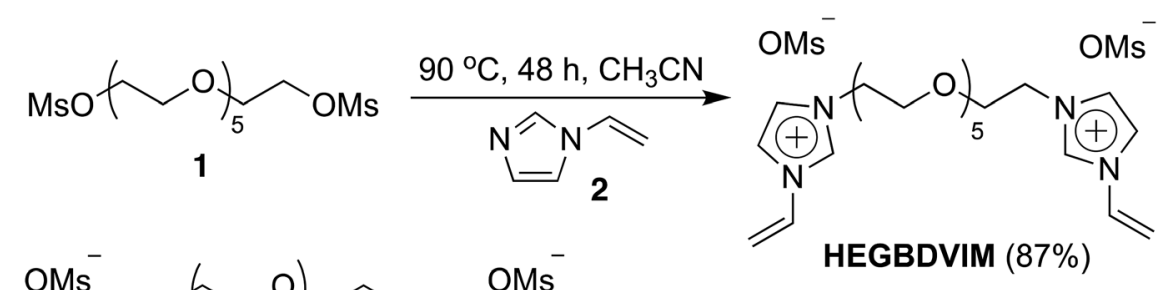

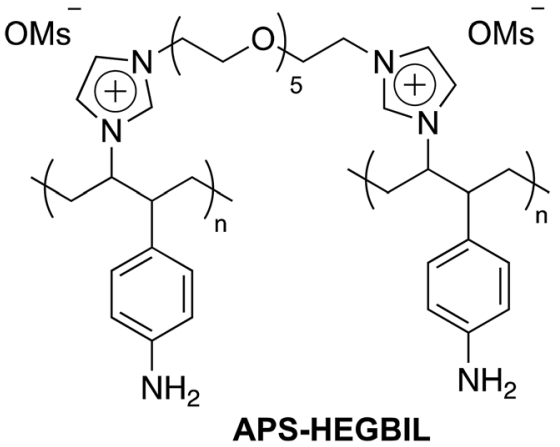

AIBN, $\mathrm{CH}_{2} \mathrm{Cl}_{2}, 70^{\circ} \mathrm{C}, 24 \mathrm{~h}$

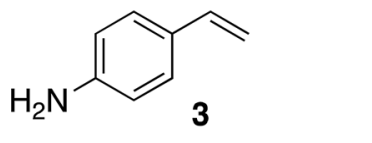

Scheme 1 Preparation of APS-HEGBIL.

synthetic targets in the pharmaceutical field. While the synthesis of alcohols from alkyl halides by nucleophilic substitution has a long history, ${ }^{19}$ simple replacement of halogens by water is difficult due to its poor nucleophilicity under mild alkaline conditions. ${ }^{\mathbf{2 0}-22}$ Therefore, various methods have been devised to transform halo compounds into alcohols under homogeneous catalytic conditions via $\mathrm{S}_{\mathrm{N}^{2}}$ type reactions in the presence or absence of strong bases. ${ }^{23-30}$ However, these procedures suffer from the need for large amounts of chemical catalysts, requirements for high boiling solvents and long reaction times, the formation of by-products, and difficulties associated with separating homogeneous catalysts from reaction media. The eco-friendly, nucleophilic hydroxylation of halide compounds using aqueous ILs (Fig. 1) was recently reported by our group. ${ }^{30,31}$ However, this methodology also suffers from long reaction times, the requirement for excessive amounts of ILs, and difficulties separating ILs from reaction media. Therefore, the development of a truly recyclable heterogeneous catalytic process that uses green reaction conditions would represent a significant advancement.

In this work, by taking advantage of the above described merits of PSILs, we developed an efficient and simple protocol for preparing an amino-polystyrene supported hexaethylene glycol-bridged ionic liquid (APS-HEGBIL) by using a crosslinking polymerization reaction between hexaethylene glycolbridged dicationic 1-vinyl imidazole salt (HEGBDVIM) and amino-styrene. The as-synthesized APS-HEGBIL material was used as a heterogeneous catalyst for the nucleophilic hydroxylation of alkyl halides in water and resulting in corresponding alcohol products. As a result of polymer-support and the use of flexible imidazolium linkers, the catalyst produced acted as an efficient "quasi-homogeneous" catalyst with high activity and selectivity, but was easily isolated and reused like heterogeneous catalysts. As these goals were accomplished on the surface of a polymer, this novel catalyst represents a significant bridge between homogeneous and heterogeneous catalysis.

\section{Results and discussion}

APS-HEGBIL was synthesized as shown in Scheme 1. First, HEGBDVIM was prepared by $N$-alkylation of vinyl imidazole 2 with hexaethylene glycol dimesylate 1 . Cross-linking polymerization between HEGBDVIM and amino styrene 3 generated APSHEGBIL, which was characterized by elemental analysis (EA), FT-IR, solid state NMR, XPS, and TGA. Based on EA analysis of sulfur, APS-HEGBIL was found to contain approximately $1.2 \mathrm{mmol}$ per gram of the imidazolium mesylate moiety.

A comparison of the FT-IR spectra of APS-HEGBIL and HEGBDVIM (Fig. 2) revealed the disappearance of terminal alkene $(=\mathrm{C}-\mathrm{H})$ stretch and of bending vibrations at 3137-3084, 1577 , and $1547 \mathrm{~cm}^{-1}$, which confirmed APS-HEGBIL formation. All remaining absorption peaks, including those of imidazole ring $\mathrm{C}=\mathrm{N}$ and mesylate $\mathrm{S}=\mathrm{O}$ and $\mathrm{S}-\mathrm{O}$ stretching vibrations were unchanged.

Chemical bonding in APS-HEGBIL and HEGBDVIM were investigated by X-ray photoelectron spectroscopy (XPS) (Fig. 3).

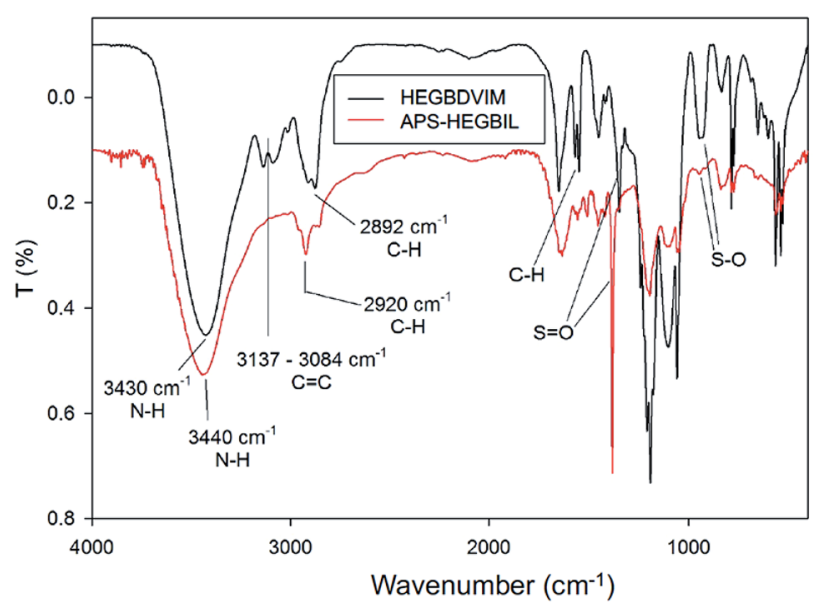

Fig. 2 FT-IR spectra of APS-HEGBIL and HEGBDVIM. 
(a)

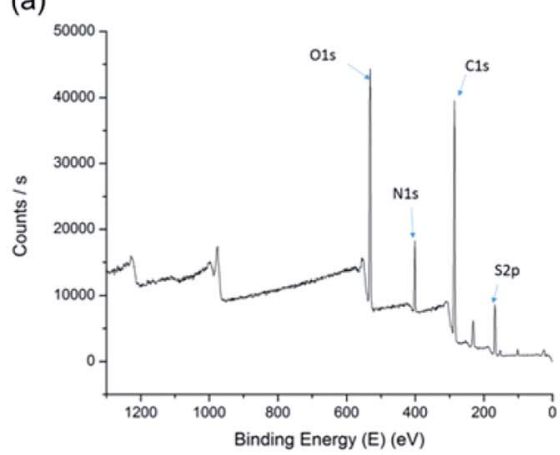

(d)

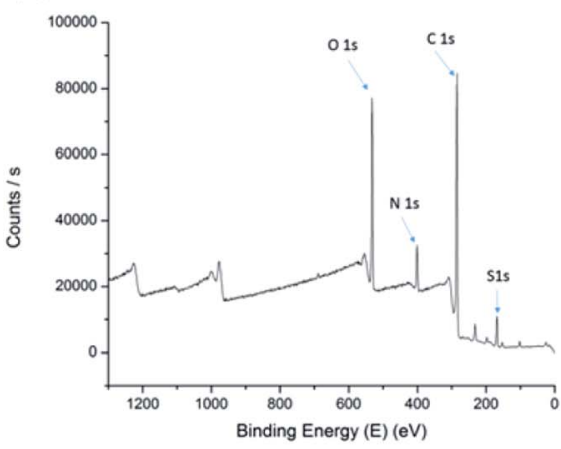

(b)

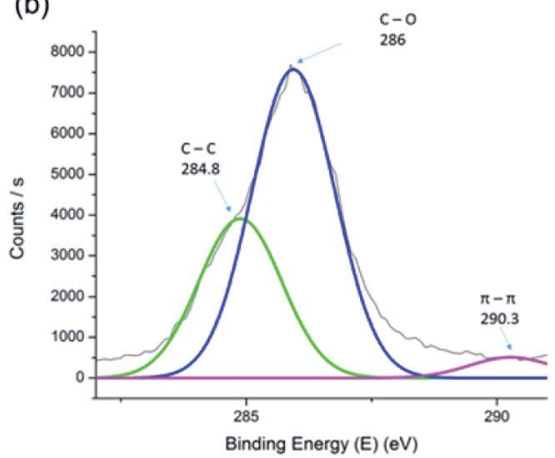

(e)

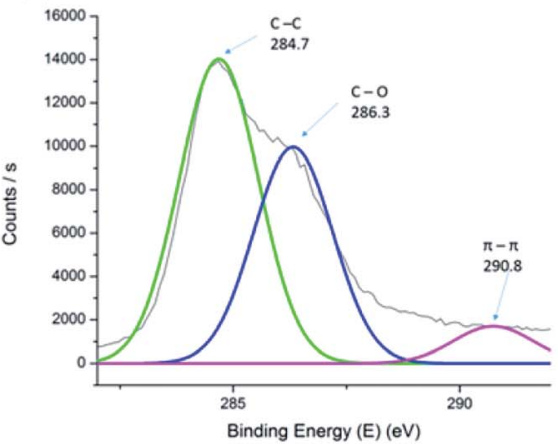

(c)

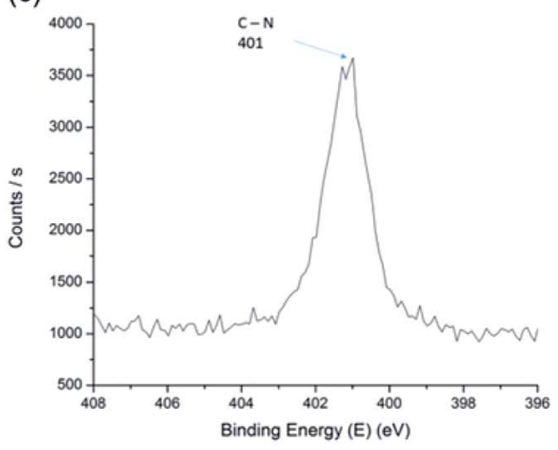

(f)

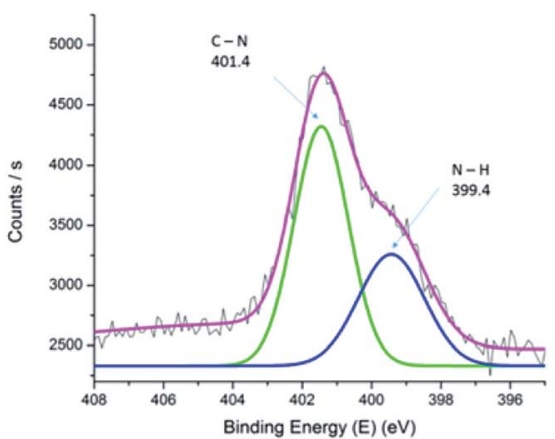

Fig. 3 XPS patterns of $(a-c)$ HEGBDVIM and (d-f) APS-HEGBIL.

Elemental peaks were dominated by $\mathrm{O}, \mathrm{C}, \mathrm{S}$, and $\mathrm{N}$ of HEGBDVIM and APS-HEGBIL (Fig. 3a and d). Noticeably, the peak intensity of $\mathrm{C}$ was almost double that of $\mathrm{N}$ in HEGBDVIM (Fig. 3a). However, after polymerization, C peak intensity was three times greater than that of N. Moreover, both HEGBDVIM and APS-HEGBIL contained the same $\mathrm{C}$ 1s peaks at 284.7$284.8 \mathrm{eV}$ (C-C bonding) and at 286-286.3 eV (C-O bonding) (Fig. $3 \mathrm{~b}$ and e). Successful polymerization was also indicated by the peak intensity ratios of bonding types. Specifically, HEGBDVIM had a peak intensity at $286 \mathrm{eV}$ (C-O bonding) that was twice as high as that at 284.8 (C-C bonding), and the peak intensity of $\pi-\pi$ bonding was low. After APS-HEGBIL formation, peak intensities changed according to the number of bonds in the circuit. In particular, the $\mathrm{C}-\mathrm{C}$ peak was significantly larger than the $\mathrm{C}-\mathrm{O}$ peak, and the height of the $\pi-\pi$ bonding peak also increased due to the aromatic groups. Furthermore, $\mathrm{N} 1 \mathrm{~s}$ base peak was observed at $401 \mathrm{eV}$ (C-N bonding) in the XPS pattern of HEGBDVIM (Fig. 3c and f), whereas a new peak was observed at $399.4 \mathrm{eV}$ (indicating $\mathrm{N}-\mathrm{H}$ bond formation) was observed after reaction (Fig. 3f), showing that APS-HEGBIL had been covalently linked to HEGBDVIM.

Solid state ${ }^{13} \mathrm{C}$ NMR spectroscopy confirmed the formation of APS-HEGBIL (Fig. 4a). All aromatic and imidazolium ring carbon signals appeared between 131-126 ppm. A higher intensity signal appeared at $71.49 \mathrm{ppm}$, indicating methylene carbons were attached to the imidazolium and aromatic rings at side chains. Quaternary and tertiary carbons in the side chains of the alkyl polymer appeared between 44.9 and $41.3 \mathrm{ppm}$. Finally, the remaining methylene and methyl carbons appeared at 25.6 and $21.9 \mathrm{ppm}$, respectively.
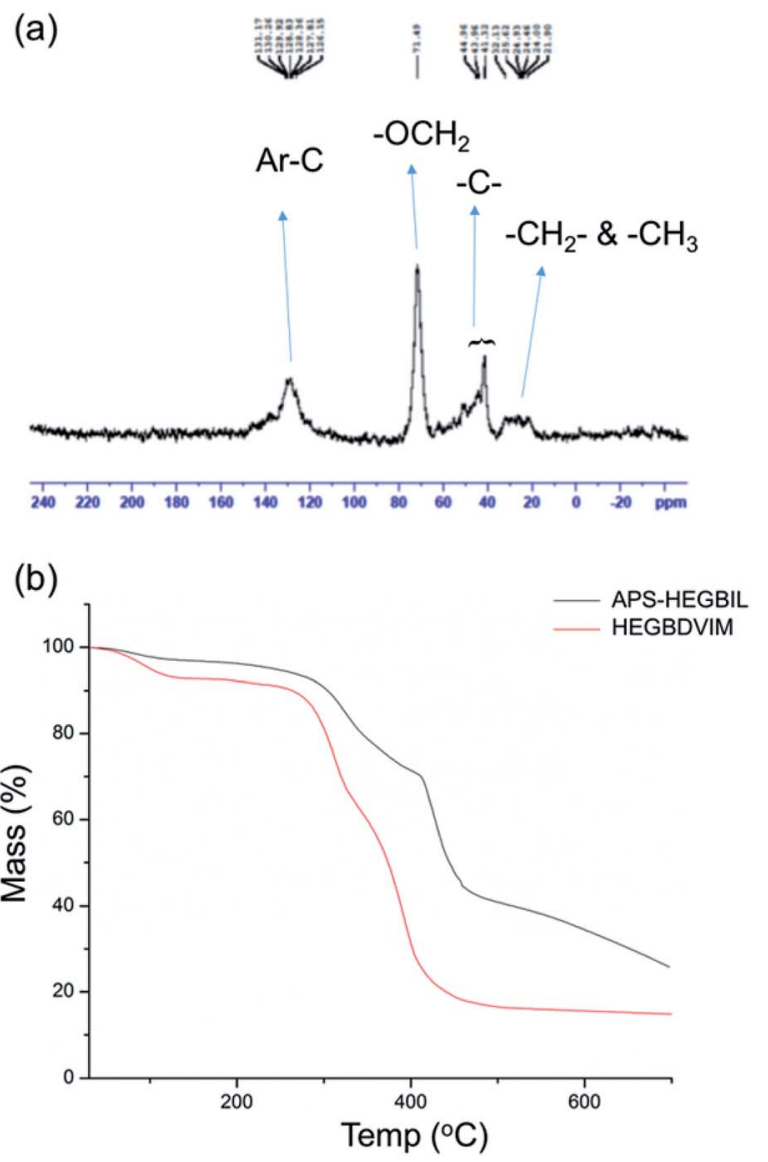

Fig. 4 (a) Solid state ${ }^{13} \mathrm{C}$ NMR spectrum of APS-HEGBIL. (b) TGA patterns of APS-HEGBIL and HEGBDVIM. 
Table 1 Hydroxylation of alkyl bromide 4 in water under various reaction conditions ${ }^{a}$

\begin{tabular}{|c|c|c|c|c|c|c|}
\hline \multirow[b]{3}{*}{ Entry } & 4 & $\begin{array}{l}\text { enger } \\
\text { source } \\
{ }^{\circ} \mathrm{C}\end{array}$ & a & & & \\
\hline & \multirow[b]{2}{*}{ Catalyst or other $\mathrm{OH}^{-}$source } & \multirow{2}{*}{$\begin{array}{l}\text { Acid scavenger } \\
\text { ( } 3 \text { equiv.) }\end{array}$} & \multirow[b]{2}{*}{ Time (min) } & \multicolumn{3}{|c|}{ Yield $^{b}$} \\
\hline & & & & 4 & $\mathbf{5 a}$ & $5 \mathbf{b}$ \\
\hline 1 & - & $\mathrm{K}_{2} \mathrm{CO}_{3}$ & 180 & 30 & 30 & 40 \\
\hline 2 & APS-HEGBIL (100 mg) & $\mathrm{K}_{2} \mathrm{CO}_{3}$ & 25 & - & 98 & Trace \\
\hline 3 & APS-HEGBIL (100 mg) & - & 65 & - & 72 & 27 \\
\hline 4 & APS-HEGBIL (100 mg) & $\mathrm{NaHCO}_{3}$ & 55 & - & 84 & 15 \\
\hline 5 & APS-HEGBIL (50 mg) & $\mathrm{K}_{2} \mathrm{CO}_{3}$ & 60 & 20 & 80 & \\
\hline 6 & APS-HEGBIL (150 mg) & $\mathrm{K}_{2} \mathrm{CO}_{3}$ & 25 & - & 98 & - \\
\hline 7 & [hexaEGmim][OMs] (1 equiv.) & $\mathrm{K}_{2} \mathrm{CO}_{3}$ & 75 & - & 90 & 10 \\
\hline 8 & [bmim $][\mathrm{OMs}]$ (1 equiv.) & $\mathrm{K}_{2} \mathrm{CO}_{3}$ & 80 & 10 & 55 & 35 \\
\hline 9 & HEGBDVIM ( 1 equiv.) & $\mathrm{K}_{2} \mathrm{CO}_{3}$ & 45 & - & 85 & 15 \\
\hline 10 & TBAOH ( 3 equiv.) & - & 65 & - & 50 & 25 \\
\hline 11 & KOH/18-crown-6 (3 equiv.) & - & 65 & 10 & 55 & 35 \\
\hline 12 & APS-HEGBIL $(100 \mathrm{mg})$ in PBS & - & 35 & - & 90 & 10 \\
\hline $13^{c}$ & APS-HEGBIL (100 mg) & $\mathrm{K}_{2} \mathrm{CO}_{3}$ & 35 & - & 98 & - \\
\hline
\end{tabular}

${ }^{a}$ All reactions were carried out on a $1.0 \mathrm{mmol}$ scale of 4 using 3 equiv. of acid scavenger or $\mathrm{OH}^{-}$source in $3.0 \mathrm{~mL}$ of water at $90{ }^{\circ} \mathrm{C} .{ }^{b} \mathrm{Yields}$ were determined by ${ }^{1} \mathrm{H}$ NMR spectroscopy. ${ }^{c}$ This reaction was performed in a solution of 1,4-dioxane $(2.0 \mathrm{~mL}) \mathrm{and} \mathrm{H}_{2} \mathrm{O}(2.0 \mathrm{~mL})$.

Thermogravimetric analysis (TGA) was used to investigate the thermal properties of APS-HEGBIL and of HEGBDVIM (Fig. 4b). The results obtained showed APS-HEGBIL was stable up to $300{ }^{\circ} \mathrm{C}$ (a high decomposition temperature for an ionic catalyst). On the other hand, HEGBDVIM lost $10 \%$ of its mass between $65{ }^{\circ} \mathrm{C}$ and $275{ }^{\circ} \mathrm{C}$ due to mesylate anion loss, and a further substantial weight loss (accounting for $80 \%$ of weight loss) was observed between 275 and $410{ }^{\circ} \mathrm{C}$, due to decomposition of imidazole groups. According to the thermograms obtained, thermal decomposition of APS-HEGBIL occurred in four stages. Between $115{ }^{\circ} \mathrm{C}$ and $300{ }^{\circ} \mathrm{C}$, it slowly lost approximately $5 \%$ of its weight, presumably due to loss of trapped moisture, from $300{ }^{\circ} \mathrm{C}$ to $412{ }^{\circ} \mathrm{C}$, it lost $25 \%$ due to mesylate decomposition, from $412{ }^{\circ} \mathrm{C}$ to $470{ }^{\circ} \mathrm{C}$, its lost $28 \%$ due to imidazole decomposition, and at temperatures above $470{ }^{\circ} \mathrm{C}$, it lost $17 \%$ due to high molecular weight polymer.

We investigated the catalytic efficacy of APS-HEGBIL for the nucleophilic hydroxylation of alkyl halides in water, which was used as a hydroxide source, under different reaction conditions. Initially, we performed the hydroxylation reaction using hexaethylene glycol (hexaEG) bromide $\mathbf{4}$ as a model substrate (Table 1). When $\mathrm{K}_{2} \mathrm{CO}_{3}$ was used in the absence of APS-HEGBIL in water at $90{ }^{\circ} \mathrm{C}$, the hydroxylation of $\mathbf{4}$ was incomplete at $180 \mathrm{~min}$, and the alkene by-product $\mathbf{5 b}(40 \%)$ was produced by $\beta$-elimination (entry 1 ). The same reaction in the presence of $100 \mathrm{mg}$ of APS-HEGBIL ( $\sim 0.12$ equiv. of the IL portion) under the same reaction conditions affording the desired alcohol 5a in $98 \%$ yield within $30 \mathrm{~min}$ (entry 2). On the other hand, in the presence of APS-HEGBIL by without $\mathrm{K}_{2} \mathrm{CO}_{3} \mathbf{5 a}$ was obtained at a yield of only $72 \%$ with the alkene by-product $5 \mathbf{b}$ ( $27 \%$, entry 3$)$. When the reaction was conducted with APS-HEGBIL in the presence of $\mathrm{NaHCO}_{3}$, the rate of the reaction was slow and a 5a was obtained in moderate yield (Table 1, entry 4). Given that $\mathrm{K}_{2} \mathrm{CO}_{3}$ was found to be the most efficient acid scavenger for this catalytic system, it was used in subsequent experiments. When the amount of APS-HEGBIL was reduced, 5a yields decreased

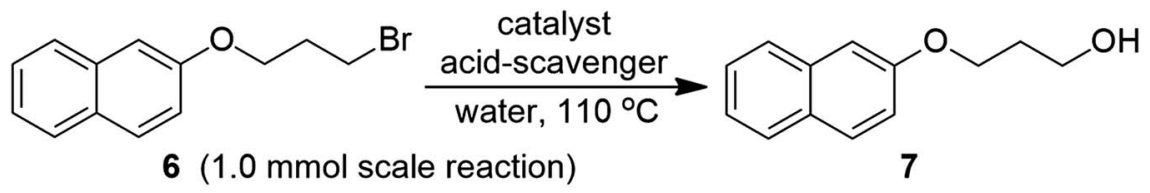

\begin{tabular}{llrr} 
catalyst (amount) & acid-scavenger & time & yield \\
\hline [bmim][BF $](4 \mathrm{~mL})$ in water/1,4-dioxane [31] & $\mathrm{NaHCO}_{3}$ & $20 \mathrm{~h}$ & $95 \%$ \\
hexaEG-DHIM (1.1 g, 1.0 eqiuv) [32] & $\mathrm{K}_{2} \mathrm{CO}_{3}$ & $5 \mathrm{~h}$ & $98 \%$ \\
APS-HEGBIL (100 mg, 0.12 equiv of IL portion) & $\mathrm{K}_{2} \mathrm{CO}_{3}$ & $2 \mathrm{~h}$ & $98 \%$
\end{tabular}

Scheme 2 Comparison of the activity of APS-HEGBIL as a heterogeneous promoter with homogenous custom-made IL promoters. 
Table 2 Synthesis of various alcohols from alkyl halides using APS-HEGBIL ${ }^{a}$

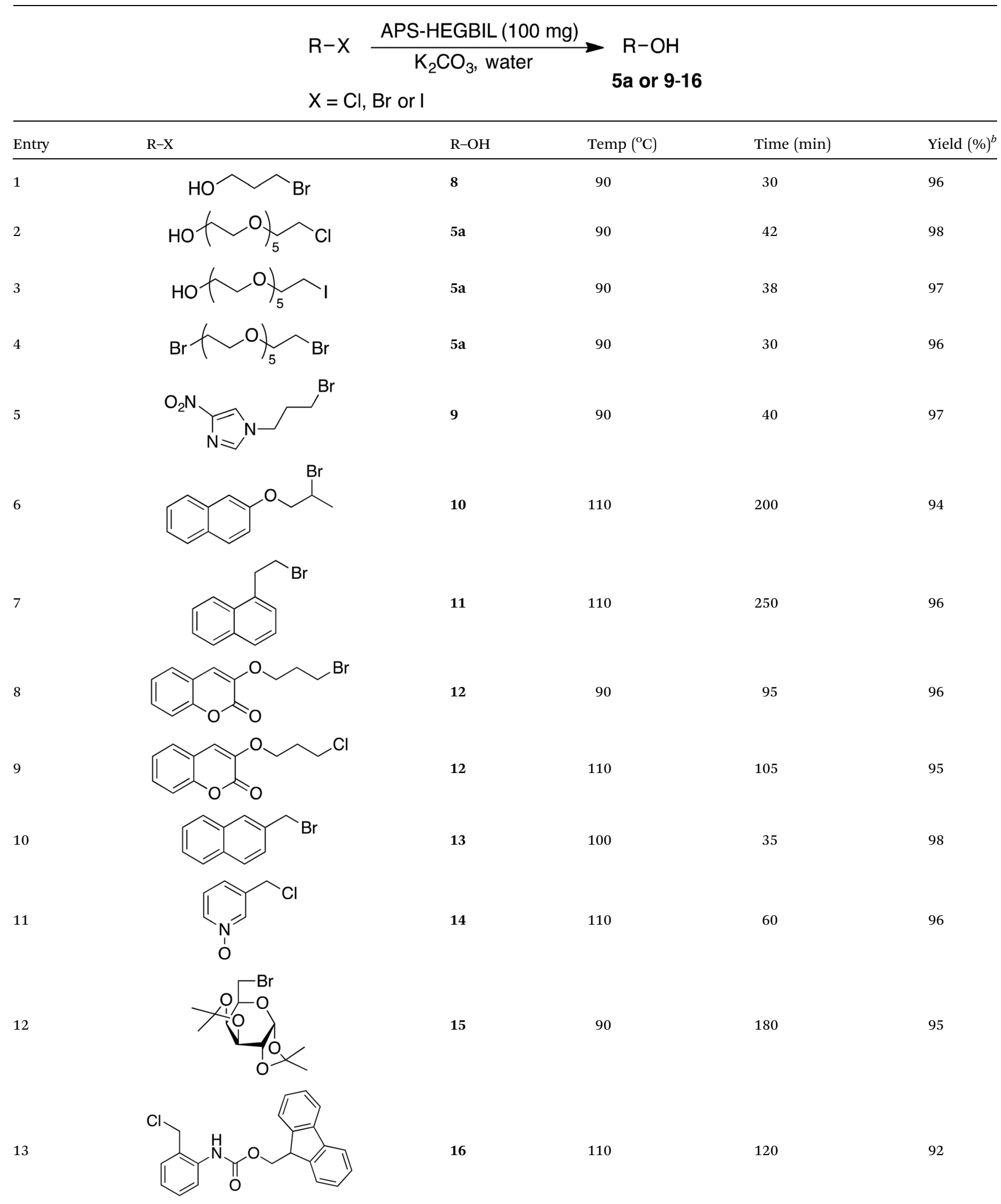

${ }^{a}$ All reactions were performed on a $1.0 \mathrm{mmol}$ scale of the substrate in the presence of APS-HEGBIL $(100 \mathrm{mg})$ and 3.0 equiv. of $\mathrm{K}_{2} \mathrm{CO}_{3}$ in $3.0 \mathrm{~mL}$ of water. ${ }^{b}$ Isolated yield. 
and reaction times increased (entry 5), whereas increasing the amount of catalyst beyond did not substantially increase yield (entry 6). To evaluate the catalytic activity and other characteristics of APS-HEGBIL, we compared it with other ILs. Initially, we performed the above hydroxylation reaction in the presence of the conventional ILs (1.0 equiv.) [hexEGmim][OMs] or [bmim $][\mathrm{OMs}]$ in water at $90{ }^{\circ} \mathrm{C}$, but found the reaction sluggish and that $5 \mathbf{a}$ was obtained in only moderate yield after prolonged reaction with alkene $\mathbf{5 b}$ (entries 7 and 8, respectively). When hydroxylation was performed using 1 equiv. of HEGBDVIM under the same reaction conditions, the reaction was slightly faster than for [hexEGmim] [OMs] or [bmim][OMs], but the elimination side reaction still occurred (entry 9). When the reaction was conducted in the presence of the conventional phase-transfer catalytic (PTC) reagents, TBAOH or $\mathrm{KOH} / 18$ crown-6 complex, reaction times were prolonged and $\mathbf{5 b}$ was obtained at higher yields (entries 10 and 11, respectively). Hydroxylation without $\mathrm{K}_{2} \mathrm{CO}_{3}$ in phosphate-buffered saline (PBS) provided 5a in 90\% yield (entry 12). The model reaction in organic/aqueous co-solvents, such as 1,4-dioxane with water (1:1) and APS-HEGBIL at $90{ }^{\circ} \mathrm{C}$ afforded the expected product in excellent yield (entry 13). However, from an environmental point of view, this method was clearly inferior to reaction in water in the presence of $\mathrm{K}_{2} \mathrm{CO}_{3}$ (Table 1, entry 2). Hence, this protocol was found to be efficient and straightforward and to enable catalyst recovery.

In addition, to the observed merits of APS-HEGBIL in comparison with reported conventional ILs, we investigated hydroxylation reactions of the nonpolar 2-(3-bromopropoxy) naphthalene (6). The results obtained demonstrated the catalytic superiority of APS-HEGBIL in Scheme 2. In previous studies on IL such as [bmim][BF4] and hexaEG-DHIM, these homogeneous catalysts were used, which were problematic with respect to catalyst purification, the use of volatile organic solvents, and required expensive catalysts at high loadings. By contrast, APSHEGBIL was easily recovered by filtration. In addition, even smaller amounts of APS-HEGBIL showed excellent performance as compared with conventional homogeneous ILs for the hydroxylation of $6 .^{31,32}$ These results suggest the nucleophilicity of water might be enhanced by the imidazolium moiety of APSHEGBIL due to PTC effect. ${ }^{32}$ The basic nature of the aniline amino groups of APS-HEGBIL inhibits the reverse reaction through reduction of acidity of the reaction medium from in situ generated $\mathrm{HBr}$. In addition, the uniform distribution of active cavities along the wrinkled structure of the catalyst, provides a "site-specific effect" on the surfaces of the catalyst." The recovery and reusability of a catalyst is an important factor from a green chemistry perspective. Therefore, we studied the reusability of APS-HEGBIL in the model reaction. After completion the reaction, APS-HEGBIL was separated by simple filtration, and we found it could be reused ten times over without appreciable loss in catalytic activity. After each recovery cycle, the reaction product 7 was obtained in excellent yield (90-98\%) (Table S1 in ESI $\dagger$ ).

Having optimized reaction conditions, we evaluated the hydroxylations of a variety of alkyl halides (Table 2). Most of the reactions proceeded efficiently and provided desired products in excellent yields. Hydroxylations of 3-bromopropanol, hexaEG chloride, hexaEG iodide, hexaEG dibromide, and 4nitroimidazole-1-propyl bromide proceeded smoothly to provide the corresponding alcohols (8, 5a or 9) in excellent yields (96, 98, 97, 96, and 97\%, entries 1-5, respectively), which demonstrated the APS-HEGBIL/water system offers a useful means of synthesizing polar alcohols. Generally, the hydroxylations of base-sensitive halides using a basic hydroxide proceed with difficulty because of a tendency to undergo elimination to form alkenes. Furthermore, APS-HEGBIL enabled the selective hydroxylations of base sensitive substrates like sec-alkyl bromide and bromoethyl aromatic compounds to corresponding alcohols (10 and 11) at high yields (94 and 96\%, entries 6 and 7 , respectively). In addition, the biologically active 3-(3bromopropoxy)-2H-chromen-2-one and 3-(3-chloropropoxy)- $2 H$ chromen-2-one gave excellent yields of 3-(3-hydroxypropoxy)$2 H$-chromen-2-one (12) even after shorter reaction times (entries 8 and 9, respectively). Bromo-sugar and 3-chloro-picoline- $N$-oxide were also converted to the corresponding alcohols in high yields with shorter reaction times (entries 11 and 12, respectively).

As a final example, a good yield of $N$-Fmoc-2hydroxymethylaniline $\mathbf{1 6}$ was obtained from the highly base sensitive substrate $N$-Fmoc-2-chloromethylaniline in water at $110{ }^{\circ} \mathrm{C}$ for $2 \mathrm{~h}$ (entry 13). While the extremely base sensitive Fmoc group can easily be lost under basic conditions, such as $\mathrm{KOH} / 18$-crown-6 in $\mathrm{CH}_{3} \mathrm{CN},{ }^{32}$ this methodology avoided cleavage of the Fmoc group and resulted in a good yield of alcohol with minimal Fmoc deprotection. These results show that this protocol provides required alcohol products rapidly in high yields even from non-polar substrates and extremely basesensitive raw materials, avoiding the productions of side products.

\section{Conclusions}

In conclusion, a highly efficient, air stable, and recyclable APSHEGBIL catalyst was designed and prepared that functioned well as a multifunctional heterogeneous catalyst for the nucleophilic hydroxylation of halides in aqueous medium. This APSHEGBIL catalytic system enhanced the nucleophilicity of water and the catalyst was easily recovered by simple filtration and reused several times without significant loss in catalytic activity. The described protocol provided alcohols in excellent yields from polar, non-polar, and even base-sensitive alkyl halide substrates. Furthermore, were believe the protocol encompasses the requirements of green chemistry as it generates few side products, is cost effective, requires non-volatile solvents, is eco-benign, and reduces the environmental toxic chemical burden. We hope the described methodology opens new routes to the development of eco-friendly, sustainable hydroxylation.

\section{Experimental}

\section{General remarks}

Unless otherwise noted, all reagents and solvents were obtained commercially. Reaction progress was followed by TLC on 
$0.25 \mathrm{~mm}$ silica gel glass plates containing F-254 indicator by visualization under UV light (254 nm). Flash chromatography was performed using 230-400 mesh silica gel. ${ }^{1} \mathrm{H}$ and ${ }^{13} \mathrm{C}$ NMR spectra were recorded on a $400 \mathrm{MHz}$ spectrometer, and chemical shifts were reported in $\delta$ units (ppm) relative to tetramethylsilane. FT-IR spectra were obtained using a Bruker Vertex 80v FT-IR spectrometer. Low- and high-resolution electron impact (EI, $70 \mathrm{eV}$ ) spectra were obtained using a high resolution mass spectrometer (Korea Basic Science Institute). X-ray photoelectron spectroscopy (XPS) measurements were performed using an angle-resolved X-ray photoelectron spectrometer (Theta Probe AR-XPS, Thermo Fisher Scientific, U.K.) equipped with an MXR1 Gun $400 \mu \mathrm{m} 15 \mathrm{keV}$ spectrometer. Thermogravimetric analysis (TGA) was performed using a TG $209 \mathrm{~F} 3$ unit (NETZSCH, Germany) at a $5{ }^{\circ} \mathrm{C} \mathrm{min}^{-1}$ heating rate between 10 and $800{ }^{\circ} \mathrm{C}$ under an argon atmosphere. Elemental analysis was performed using a FLASH EA 1112 Elemental Analyzer (Thermo Electron Corporation).

\section{Preparation of $3,3^{\prime}$-(3,6,9,12,15-pentaoxaheptadecane-1,17-diyl) bis(1-vinyl-1H-imidazol-3-ium) methanesulfonate (HEGBDVIM)}

1-Vinyl-1 $H$-imidazole $(376 \mathrm{mg}, 4.00 \mathrm{mmol}$ ) was added dropwise to a solution of $876 \mathrm{mg}(2.00 \mathrm{mmol})$ of hexaethylene glycol dimesylate in dried $\mathrm{CH}_{3} \mathrm{CN}(50 \mathrm{~mL})$. The reaction mixture was stirred at $90{ }^{\circ} \mathrm{C}$ for $48 \mathrm{~h}$. The reaction mixture was then concentrated by rotary evaporation; the concentrated mixture was washed several times with ethyl acetate $(75 \mathrm{~mL})$ and dried under high vacuum overnight at room temperature to afford $1.04 \mathrm{~g}(1.75 \mathrm{mmol}, 87 \%)$ of HEGBDVIM as a pale yellow solid. ${ }^{1} \mathrm{H}$ NMR (400 MHz, DMSO- $\left.d_{6}\right) \delta 9.42(\mathrm{t}, J=1.3 \mathrm{~Hz}, 2 \mathrm{H}), 8.20(\mathrm{t}, J=$ $1.8 \mathrm{~Hz}, 2 \mathrm{H}), 7.88(\mathrm{t}, J=1.6 \mathrm{~Hz}, 2 \mathrm{H}), 7.32(\mathrm{q}, J=8.2 \mathrm{~Hz}, 2 \mathrm{H}), 5.98$ $(\mathrm{d}, J=2.3 \mathrm{~Hz}, 1 \mathrm{H}), 5.94(\mathrm{~d}, J=2.3 \mathrm{~Hz}, 1 \mathrm{H}), 5.44(\mathrm{dd}, J=8.7$, $2.3 \mathrm{~Hz}, 2 \mathrm{H}), 4.38(\mathrm{~d}, J=5.0 \mathrm{~Hz}, 4 \mathrm{H}), 3.79(\mathrm{t}, J=4.8 \mathrm{~Hz}, 4 \mathrm{H}), 3.56-$ $3.47(\mathrm{~m}, 16 \mathrm{H}), 2.32(\mathrm{~s}, 6 \mathrm{H}) ;{ }^{13} \mathrm{C}$ NMR $\left(100 . \mathrm{MHz}, \mathrm{DMSO}-d_{6}\right) \delta$ : 135.7, 128.6, 123.5, 118.6, 108.8, 69.4, 67.8, 49.0, 39.0. MS (ESI) $m / z 594\left(\mathbf{M}^{+}+\mathrm{H}\right)$; HRMS (ESI) $m / z$ calcd for $\mathrm{C}_{24} \mathrm{H}_{42} \mathrm{~N}_{4} \mathrm{O}_{9} \mathrm{~S}_{2}\left(\mathbf{M}^{+}+\right.$ H) 594.2393, found 594.2390.

\section{Preparation of APS-HEGBIL}

To a mixture of $p$-aminostyrene (1.8 g), HEGBDVIM (594 mg), and dry $\mathrm{CH}_{2} \mathrm{Cl}_{2}(20 \mathrm{~mL})$ in a $50 \mathrm{~mL}$ round bottom flask was added $30 \mathrm{mg}$ of AIBN. The reaction mixture was then stirred for $24 \mathrm{~h}$ at $70{ }^{\circ} \mathrm{C}$ under $\mathrm{N}_{2}$, and the product so obtained was precipitated from diethyl ether and washed several times with acetone and methanol. The APS-HEGBIL catalyst was then dried under vacuum at $40{ }^{\circ} \mathrm{C}$ overnight. Anal.; N 8.2, C 60.4, H 7.7, S $4.0\left(1.2 \mathrm{mmol} \mathrm{S} \mathrm{g}^{-1}\right)$.

\section{Typical procedure for nucleophilic hydroxylation in Table 1} (entry 2)

APS-HEGBIL (100 mg) was added to a mixture of 17-bromo3,6,9,12,15-pentaoxaheptadecan-1-ol (4, $344 \mathrm{mg}, 1.0 \mathrm{mmol})$ and $\mathrm{K}_{2} \mathrm{CO}_{3}(415 \mathrm{mg}, 3 \mathrm{mmol})$ in water $(3 \mathrm{~mL})$, and the reaction was stirred at $90{ }^{\circ} \mathrm{C}$ for $25 \mathrm{~min}$. After reaction completion (confirmed by TLC), the mixture was cooled to room temperature, diethyl ether was added, the reaction mixture was filtered, and the filtrate was evaporated under reduced pressure. Flash column chromatography ( $5 \%$ methanol/dichloromethane) of the filtrate afforded $278 \mathrm{mg}(0.98 \mathrm{mmol}, 98 \%)$ of hexaethylene glycol (5a) as a colorless oil. ${ }^{1} \mathrm{H}$ NMR $\left(400 \mathrm{MHz}, \mathrm{CDCl}_{3}\right) \delta 3.68$ (d, $J=11.0 \mathrm{~Hz}, 4 \mathrm{H}), 3.62(\mathrm{~s}, 17 \mathrm{H}), 3.59-3.54(\mathrm{~m}, 4 \mathrm{H}), 2.59(\mathrm{~s}, 2 \mathrm{H})$; ${ }^{13} \mathrm{C}-\mathrm{NMR}\left(100 \mathrm{MHz}, \mathrm{CDCl}_{3}\right) \delta 72.4,70.3,70.2,70.0,61.3$; MS (EI) $m / z 283.1\left(\mathrm{M}^{+}\right)$; HRMS (EI TOF) $m / z$ calcd for $\mathrm{C}_{12} \mathrm{H}_{26} \mathrm{O}_{7}\left(\mathrm{M}^{+}\right)$ 283.1757, found 283.1759.

\section{Conflicts of interest}

There are no conflicts to declare.

\section{Acknowledgements}

This work was supported by an Inha University Research Grant (56909).

\section{Notes and references}

1 P. Anastas and N. Eghbali, Chem. Soc. Rev., 2010, 39, 301312.

2 S. Sobhani, F. Zarifi and J. Skibsted, ACS Sustainable Chem. Eng., 2017, 5, 4598-4606.

3 J. Zhou, Y. Li, H.-B. Sun, Z. Tang, L. Qi, L. Liu, Y. Ai, S. Li, Z. Shaob and Q. Liang, Green Chem., 2017, 19, 3400-3407.

4 P. Xu, G. W. Zheng, P. X. Du, M. H. Zong and W. Y. Lou, ACS Sustainable Chem. Eng., 2016, 4, 371-386.

5 V. H. Jadhav, J. G. Kim, S. H. Park and D. W. Kim, Chem. Eng. J., 2017, 308, 664-668.

6 K. Matuszek, A. Chrobok, P. Latos, M. Markiton, K. Szymanska, A. Jarzębskibc and M. S. Kwasny, Catal. Sci. Technol., 2016, 6, 8129-8137.

7 A. Weiß, M. Munoz, A. Haas, F. Rietzler, H. P. Steinruck, M. Haumann, P. Wasserscheid and B. J. M. Etzold, ACS Catal., 2016, 6, 2280-2286.

8 D. W. Kim and D. Y. Chi, Angew. Chem., Int. Ed., 2004, 43, 483-485.

9 D. W. Kim, D. J. Hong, K. S. Jang and D. Y. Chi, Adv. Synth. Catal., 2006, 348, 1719-1727.

10 W. Qian, J. Texter and F. Yan, Chem. Soc. Rev., 2017, 46, 1124-1159.

11 W. Wang, C. Li, L. Yan, Y. Wang, M. Jiang and Y. Ding, ACS Catal., 2016, 6, 6091-6100.

12 X. Mu, J. Meng, Z. Li and Y. Kou, J. Am. Chem. Soc., 2005, 127, 9694-9695.

13 Y. Xie, Z. Zhang, T. Jiang, J. He, B. Han, T. Wu and K. Ding, Angew. Chem., Int. Ed., 2007, 46, 7255-7258.

14 F. Messina and O. Rosati, Curr. Org. Chem., 2013, 17, 11581178.

15 Y. Gu, Green Chem., 2012, 14, 2091-2128.

16 J. Niemeier, R. V. Engela and M. Rose, Green Chem., 2017, 19, 2839-2845.

17 G. Brahmachari, ACS Sustainable Chem. Eng., 2015, 3, 23502358.

18 M. B. Gawande, V. D. B. Bonifacio, R. Luque, P. S. Branco and R. S. Varma, Chem. Soc. Rev., 2013, 42, 5522-5551. 
19 M. Harris and M. J. Bull, Synth. Commun., 1985, 15, 12251228.

20 D. W. Kim, D.-S. Ahn, Y.-H. Oh, S. Lee, H. S. Kil, S. J. Oh, S. J. Lee, J. S. Kim, J. S. Ryu, D. H. Moon and D. Y. Chi, J. Am. Chem. Soc., 2006, 123, 16394-16397.

21 M. D. Smith and J. March, Advanced Organic Chemistry, Wiley and Sons, New York, 5th edn, 2001, pp. 462-474.

22 M. Makosza and K. Wojciechowski, Chem. Rev., 2004, 104, 2631-2666.

23 R. C. Larock, Comprehensive Organic Transformations, VCH, New York, 1986.

24 R. A. Johnson and E. G. Nidy, J. Org. Chem., 1975, 40, 16781680.

25 E. V. Dehmlow and S. S. Dehmlow, Phase Transfer Catalysis, VCH, New York, 3rd edn, 1993.
26 R. O. Hutchins and I. M. Taffer, J. Org. Chem., 1983, 48, 13601362.

27 L. Liu, J. A. Henderson, A. Yamamoto, P. Bremond and Y. Kishi, Org. Lett., 2012, 14, 2262-2265.

28 C. Reichardt and T. Welton, Solvents and Solvent Effects in Organic Chemistry, Wiley-VCH, Weinheim, Germany, 4th edn, 2011.

29 B. M. Chougala, S. Samundeeswari, M. Holiyachi and L. A. Shastri, ChemistrySelect, 2017, 2, 1290-1296.

30 J. H. Lee, U. Sirion, K. S. Jang, B. S. Lee and D. Y. Chi, Bull. Korean Chem. Soc., 2008, 29, 2491-2495.

31 D. W. Kim, D. J. Hong, J. W. Seo, H. S. Kim, H. K. Kim, C. E. Song and D. Y. Chi, J. Org. Chem., 2004, 69, 3186-3189. 32 V. H. Jadhav, J. G. Kim, H. J. Jeong and D. W. Kim, J. Org. Chem., 2015, 80, 7275-7280. 